\title{
Olech's correction to the proof of his theorem on existence of solutions to differential inclusions
}

\author{
Tadeusz Rzeżuchowski ${ }^{1}$
}

Received: 28 December 2018 / Accepted: 29 April 2019 / Published online: 23 May 2019

(c) The Author(s) 2019

\begin{abstract}
In 1975 Czeslaw Olech published in this journal a theorem on existence of solutions to differential inclusions with right-hand side measurable with respect to the time variable, upper semicontinuous with respect to the state variable and continuous at the arguments at which it is not convex. It occurred that the proof contained errors. This paper relates its correct version with some simplifications.
\end{abstract}

Keywords Differential inclusions · Existence of solutions · Mixed assumptions · Measurability $\cdot$ Semicontinuity $\cdot$ Continuity

Mathematics Subject Classification 34A60 · 34A12

\section{Introduction}

In 1975 appeared paper [10] by Czeslaw Olech. It unified two earlier versions of theorems on existence of solutions to differential inclusions $\dot{x} \in F(t, x)$ with $F(t, x) \subset \mathbb{R}^{d}$. Namely, the case of $F(t, x)$ convex with upper semicontinuous dependence on $x$, the other one for $F(t, x)$ only compact but continuous in $x$. The first one was in its essence known since the papers by Marchaud [9] and Zaremba [13] (formulated in a different language) and later on by many other authors. The problem for nonconvex $F(t, x)$, posed in [5], was first solved by Filippov in [3] for $F$ continuous with respect to the joint variable $(t, x)$, next by Kaczyński and Olech in [6] for $F$ continuous in $x$ and measurable in $t$.

The theorem presented in [10] contained the case of $F$ upper semicontinuous in $x$ and continuous at those $x$ at which the set $F(t, x)$ is not convex (plus measurability in $t$ ). The proof was partly based on the extension of methods introduced in [3].

While studying [10], about 1978, I remarked that some statements relating to measurability were not true. I communicated it to the Author. After a few weeks he handed me a typewritten text entitled Correction to the paper "Existence of Solutions of Non-Convex Orientor Fields".

Tadeusz Rzeżuchowski

t.rzezuchowski@mini.pw.edu.pl

1 Faculty of Mathematics and Information Science, Warsaw University of Technology, Koszykowa 75, 00-662 Warsaw, Poland 
As I was very busy then, mainly with my own thesis, I have only glanced at it and took it for granted that the problem was solved and the Author would send the correction to the journal. I lived in that conviction till 2016 when former students of Professor Olech decided to organize a conference to honor his memory-he deceased in July 2015. I planned to give a talk devoted to the Theorem from [10] and started to look for the published version of the correction. To my great astonishment I did not find it and it seems almost certain that it did not appear.

During my talk some Colleagues, friends of late Professor Olech, suggested that I should prepare for publication a corrected version of the proof. While analyzing the manuscript of the correction, which I have fortunately kept, I realized that this text is rather far from ready to go to print. Moreover, even if published (after some improvements) it would make understanding the whole proof a bit difficult as a potential reader would have to do for himself a synthesis of both papers. So I decided that the best way would be to rework the whole proof and present it in one, unified version-that is the goal of the present paper.

While working on the text I realized that it can be shortened due to some results known at the time of publication of [10] but may be not easily accessible. They were gathered in [2] which appeared in 1976.

\section{Some necessary notions and formulation of the theorem}

The usual norm in $\mathbb{R}^{d}$ will be denoted as $|x|, d(y, U)=\inf \{|y-u|: u \in U\}$ is the distance from $y$ to the set $\mathrm{U}$ and $h(U, V)$ the Hausdorff metric in the space of closed, bounded sets.

A multifunction $\Phi: W \rightsquigarrow \mathbb{R}^{d}$ (where $W \subset \mathbb{R}^{p}$ ) with closed, bounded values is continuous if it is continuous with respect to the Hausdorff metric in the range. It is upper semicontinuous at $v \in W$ if $\forall \varepsilon>0, \exists \delta>0, \forall w \in W:|v-w|<\delta \Rightarrow \Phi(v) \subset \Phi(w)+\varepsilon \cdot B_{1}\left(B_{1}\right.$ is the unit ball and addition is understood in the sense of Minkowski).

A multifunction $\Phi: I \rightsquigarrow \mathbb{R}^{d}(I=[0,1])$ with closed values is called measurable if for every closed set $U \subset \mathbb{R}^{d}$ the set $\{t \in I: \Phi(t) \cap U \neq \emptyset\}$ belongs to the Lebesgue $\sigma$-field $\mathscr{L}$. Thanks to the Kuratowski-Ryll-Nardzewski Theorem [7] we know that every such multifunction $\Phi$ with nonempty, closed values, has a measurable, univalued selection.

The measurability of $\Phi$ with closed values is equivalent to the measurability of functions $d(y, \Phi(\cdot))$ for every $y \in \mathbb{R}^{d}$-we often put a dot at the place of a variable to denote the function of just that variable. It is also equivalent to the measurability of the graph $\operatorname{Gr}(\Phi)=$ $\left\{(t, u) \in I \times \mathbb{R}^{d}: u \in \Phi(t)\right\}$ in the $\sigma$-field $\mathscr{L} \times \mathscr{B}\left(\mathscr{B}\right.$ is the Borel $\sigma$-field in $\left.\mathbb{R}^{d}\right)$. This last condition, $\mathscr{L} \times \mathscr{B}$-measurability of $\operatorname{Gr}(\Phi)$, is used to define the measurability of multifunctions with values not necessarily closed. Due to von Neumann's Theorem we know that such multifunction with nonempty values has a measurable selection. An important property of $\mathscr{L} \times \mathscr{B}$ measurable sets is the $\mathscr{L}$-measurability of their projections on $I$. (All these properties are described in [2, Chapter III.4])

\subsection{Formulation of Olech's theorem}

Let $F$ be a multifunction defined on $I \times \mathbb{R}^{d}$ with compact values in $\mathbb{R}^{d}$. We assume that for all $t \in I$ and $x \in \mathbb{R}^{d}$ the following conditions are satisfied:

(i) $F(\cdot, x)$ is measurable.

(ii) $F(t, \cdot)$ is upper semicontinuous.

(iii) $\max \{|u|: u \in F(t, x)\} \leq \lambda(t)$ with $\lambda(\cdot) \in L^{1}$. 
(iv) If the set $F(t, x)$ is not convex then $F(t, \cdot)$ is continuous at $x$.

Theorem 1 Under assumptions (i), (ii), (iii) and (iv) the Cauchy problem

$$
\dot{x} \in F(t, x), \quad x(0)=\xi
$$

has, for every $\xi \in \mathbb{R}^{d}$, a Carathéodory solution defined on $I$.

\section{Preliminary properties}

By $B(u, r)$ and $\bar{B}(u, r)$ we denote open and closed balls in $\mathbb{R}^{d}$.

Let $K=\bar{B}\left(\xi, \int_{0}^{1} \lambda t d t\right)$ and $Z$ be some fixed, countable set dense in $K$. We define on $I \times K$ an auxiliary multifunction $\hat{F}$

$$
\hat{F}(t, x)=\bigcap_{\varepsilon>0} \operatorname{cl} \bigcup\{F(t, z): z \in Z \cap B(x, \varepsilon)\}
$$

(cl stands for the closure). Due to (ii) we have the inclusion $\hat{F}(t, x) \subset F(t, x)$. If $F(t, \cdot)$ is continuous at $x$ then $\hat{F}(t, x)=F(t, x)$. Moreover $\hat{F}(t, z)=F(t, z)$ for $z \in Z$. The multifunction $\hat{F}(t, \cdot)$ is upper semicontinuous for every $t$.

For $t \in[0,1], x \in K, y \in \mathbb{R}^{d}$, put

$$
\gamma(t, x, y)=\liminf _{Z \ni z \rightarrow x} d(y, F(t, z)) .
$$

It is simple to prove that

$$
\gamma(t, x, y)=d(y, \hat{F}(t, x)) .
$$

Proposition 1 The function $\gamma(t, x, y)$ defined by (3) is $\mathscr{L} \times \mathscr{B}$ measurable with respect to $(t, x)$ for every fixed $y$ and so the multifunction $\hat{F}$ is $\mathscr{L} \times \mathscr{B}$ measurable.

Proof For every $y \in \mathbb{R}^{d}$ and $\delta>0$ the set

$$
\{(t, z) \in I \times Z: B(y, \delta) \cap F(t, z) \neq \emptyset\}=\bigcup_{z \in Z}\{t \in I: B(y, \delta) \cap F(t, z) \neq \emptyset\} \times\{z\}
$$

is $\mathscr{L} \times \mathscr{B}$ measurable in $I \times \mathbb{R}^{d}$ thanks to (i) and countability of $Z$.

We have the equality

$$
\begin{aligned}
& \{(t, x) \in I \times K: \gamma(t, x, y) \leq \varepsilon\} \\
& \quad=\bigcap_{n \in \mathbb{N}} \operatorname{cl}_{x}\{(t, z) \in I \times Z: B(y, \varepsilon+1 / n) \cap F(t, z) \neq \emptyset\},
\end{aligned}
$$

-for $A \subset I \times \mathbb{R}^{d}$ by cl $_{x} A$ we mean the set $\bigcup_{t \in I}\left(\{t\} \times \operatorname{cl}\left\{x \in \mathbb{R}^{d}:(t, x) \in A\right\}\right)$.

Due to [2] (Theorem III.22 and III.30.c) every set in the intersection at the right-hand side of (5) is $\mathscr{L} \times \mathscr{B}$ measurable so the intersection also is. This ends the proof.

As a consequence we get the following proposition.

Proposition 2 For every fixed $y \in K$ the function

$$
(t, x) \rightarrow h(\hat{F}(t, x), \hat{F}(t, y))
$$

is $\mathscr{L} \times \mathscr{B}$ measurable. 
Proof Let $\varphi_{n}$, for $n \in \mathbb{N}$, be $\mathscr{L} \times \mathscr{B}$ measurable selections of $\hat{F}$ such that $\hat{F}(t, x)=$ $\operatorname{cl}\left\{\varphi_{n}(t, x): n \in \mathbb{N}\right\}$. The equality

$$
h(\hat{F}(t, x), \hat{F}(t, y))=\max \left\{\sup _{k \in \mathbb{N}} \inf _{l \in \mathbb{N}}\left|\varphi_{k}(t, x)-\varphi_{l}(t, y)\right|, \sup _{l \in \mathbb{N}} \inf _{k \in \mathbb{N}}\left|\varphi_{k}(t, x)-\varphi_{l}(t, y)\right|\right\}
$$

entails the assertion.

Definition 1 For $t \in I, r>0$ and $x \in K$ we put

$$
\alpha(t, x, r)=\sup \{h(\hat{F}(t, x), \hat{F}(t, y)): y \in K \cap B(x, r)\} .
$$

Proposition 3 For every fixed $r \geq 0$ the function $\alpha(t, x, r)$ is $\mathscr{L} \times \mathscr{B}$ measurable with respect to $(t, x)$.

Proof The definition of $\hat{F}$ implies that

$$
\alpha(t, x, r)=\sup \{h(\hat{F}(t, x), \hat{F}(t, z)): z \in Z \cap B(x, r)\} .
$$

and so the assertion is a consequence of Proposition 2.

We introduce now an auxiliary multifunction of one variable

$$
H(t)=\left\{x \in K: \lim _{r \rightarrow 0+} \alpha(t, x, r)=0\right\}
$$

From the definition we see that $x \in H(t)$ iff $\hat{F}(t, \cdot)$ is continuous at $x$.

Remark that the sets $H(t)$ need be neither closed nor open, however the following property is true.

Proposition 4 For every $t$ the set of $x$ for which $F(t, x)$ is not convex is open and contained in $H(t)$.

Proof If $F(t, x)$ is not convex then there are points $u_{1}, u_{2} \in F(t, x)$, and numbers $\varepsilon>0$, $\varrho \in(0,1)$ such that $B\left(\varrho_{1} u_{1}+(1-\varrho) u_{2}, 2 \varepsilon\right) \cap F(t, x)=\emptyset$. For some $\omega>0$ if $\left|v_{1}-u_{1}\right|$, $\left|v_{2}-u_{2}\right|<\omega$ then $\varrho v_{1}+(1-\varrho) v_{2} \in B\left(\varrho_{1} u_{1}+(1-\varrho) u_{2}, \varepsilon\right)$. As $F(t, \cdot)$ is continuous at $x$ there is $\delta>0$ such that if $|x-y|<\delta$ then $F(t, y) \cap B\left(\varrho u_{1}+(1-\varrho) u_{2}, \varepsilon\right)=\emptyset$ and also $F(t, y) \cap B\left(u_{1}, \omega\right) \neq \emptyset$ and $F(t, y) \cap B\left(u_{2}, \omega\right) \neq \emptyset$. The segment joining some points $v_{1}$ and $v_{2}$ from these intersections has nonempty intersection with $B\left(\varrho u_{1}+(1-\varrho) u_{2}, \varepsilon\right)$ and thus $F(t, y)$ is not convex. This, thanks to (iv), implies our assertion.

For $t \in I, x \in \mathbb{R}^{d}$ and $s>0$ we put

$$
H^{c}(t)=\mathbb{R}^{d} \backslash H(t), \quad H_{s}(t)=\left\{x: d\left(x, H^{c}(t)\right)>2 s\right\}
$$

Proposition 5 The graphs of $H, H^{c}$ and $H_{S}$ are $\mathscr{L} \times \mathscr{B}$ measurable.

Proof Due to the monotonicity of $\alpha(t, x, \cdot)$ we have

$$
\lim _{r \rightarrow 0+} \alpha(t, x, r)=\lim _{n \rightarrow \infty} \alpha(t, x, 1 / n) .
$$

The functions $(t, x) \rightarrow \alpha(t, x, 1 / n)$ are $\mathscr{L} \times \mathscr{B}$ measurable so the set of arguments for

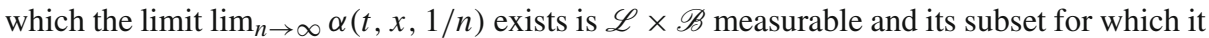
is equal 0 is also $\mathscr{L} \times \mathscr{B}$ measurable. This is exactly the graph of $H$. The graph of $H^{c}$ is its complement.

For every $t \in I$ the function $d\left(\cdot, H^{c}(t)\right)$ is continuous and for every $x \in \mathbb{R}^{d}$ the function $d\left(x, H^{c}(\cdot)\right)$ is measurable, so $d\left(x, H^{c}(t)\right)$ is $\mathscr{L} \times \mathscr{B}$ measurable with respect to $(t, x)$. The 
equality $\operatorname{Gr}\left(H_{S}\right)=\left\{(t, x): d\left(x, H^{c}(t)\right)>2 s\right\}$ entails the $\mathscr{L} \times \mathscr{B}$ measurability of this graph.

For every $t \in I$ and $r, s>0$ we put

$$
\eta(t, r, s)= \begin{cases}\sup \left\{\alpha(t, x, r): x \in H_{S}(t)\right\}, & \text { if } H_{S}(t) \neq \emptyset, \\ 0, & \text { if } H_{S}(t)=\emptyset .\end{cases}
$$

Proposition 6 For all $r, s>0$ the function $\eta(\cdot, r, s)$ is measurable and for every fixed $s>0$

$$
\lim _{r \rightarrow 0+} \int_{0}^{1} \eta(t, r, s) d t=0 .
$$

Proof For fixed $r, s>0$ and every $\varepsilon \geq 0$ the set at the right-hand side of

$$
\{t: \eta(t, r, s)>\varepsilon\}=\operatorname{proj}_{I}\left(\{(t, x): \alpha(t, x, r)>\varepsilon\} \cap \operatorname{Gr}\left(H_{S}\right)\right)
$$

is $\mathscr{L}$-measurable due to the Projection Theorem [2, Thm. III.23, page 75].

If $H_{s}(t)=\emptyset$ then $\eta(t, r, s)=0$. For $H_{s}(t) \neq \emptyset$ and $r<s$ we have

$$
\eta(t, r, s) \leq \sup \left\{h(\hat{F}(t, u), \hat{F}(t, v)): d\left(u, H^{c}(t)\right), d\left(v, H^{c}(t)\right) \geq s \text { and }|u-v|<r\right\} .
$$

The set $\left\{x: d\left(x, H^{c}(t)\right) \geq s\right\}$ is compact, so $\hat{F}(t, \cdot)$ is uniformly continuous in it and thus $\eta(t, r, s) \rightarrow 0$ when $r \rightarrow 0+$. We have also $0 \leq \eta(t, r, s) \leq 2 \lambda(t)$ with $\lambda(\cdot)$ integrable and thus (8) is true.

\section{Defining approximate solutions}

Let us fix a strictly decreasing sequence $s_{i}$ convergent to zero. Due to Proposition 6 we may also fix another sequence $r_{i}>0$ for which

$$
\sum_{i=1}^{\infty} \int_{0}^{1} \eta\left(t, r_{i}, s_{i}\right) d t<+\infty
$$

and moreover

$$
r_{i+1}<r_{i} / 2 \text {. }
$$

Let, for every $i \in \mathbb{N}, A_{i} \subset Z$ be a finite set such that $K \subset \bigcup_{a \in A_{i}} B\left(a_{i}, r_{i} / 2\right)$. $C_{n}$ will denote the set of all finite sequences $\left(a_{1}, \ldots, a_{n}\right) \in A_{1} \times \cdots \times A_{n}$ such that $\left|a_{i}-a_{i-1}\right|<r_{i-1}$ for $i=2, \ldots, n$. Obviously $C_{n}$ are nonempty and $\left(a_{1}, \ldots, a_{n}\right) \in C_{n}$ implies $\left(a_{1}, \ldots, a_{n-1}\right) \in$ $C_{n-1}$.

Using an induction argument we associate now with every $\left(a_{1}, \ldots, a_{n}\right) \in C_{n}$ a measurable function $u_{a_{1}, \ldots, a_{n}}: I \rightarrow \mathbb{R}^{d}$ in such a way that:

$$
\begin{aligned}
& u_{a_{1}, \ldots, a_{n}}(t) \in \hat{F}\left(t, a_{n}\right), \\
& \left|u_{a_{1}, \ldots, a_{n}}(t)-u_{a_{1}, \ldots, a_{n-1}}(t)\right| \leq \alpha\left(t, r_{n-1}, a_{n-1}\right) .
\end{aligned}
$$

For $n=1$ and arbitrary $a_{1} \in A_{1}$ we fix a measurable selection $u_{a_{1}}(t) \in \hat{F}\left(t, a_{1}\right)$.

Suppose that the functions $u_{a_{1}, \ldots, a_{n}}$ has been chosen for all finite sequences $\left(a_{1}, \ldots, a_{n}\right) \in$ $C_{n}$ and let us fix one. If $\left|a_{n+1}-a_{n}\right|<r_{n}$ for some $a_{n+1} \in A_{n+1}$ then the nonempty intersection $\hat{F}\left(t, a_{n+1}\right) \cap \bar{B}\left(u_{a_{1}, \ldots, a_{n}}(t)\right)$ defines a measurable multifunction and has a measurable selection $u_{a_{1}, \ldots, a_{n}, a_{n+1}}$ satisfying (11) and (12) with $n$ replaced by $n+1$ - this completes the induction argument. 
We fix now a sequence $h_{n}>0$ for which $1 / h_{n}, h_{n} / h_{n+1} \in \mathbb{N}$ and

$$
\int_{t}^{t+h_{n}} \lambda(\tau) d \tau<\frac{r_{n}}{4} \text { for } t \in\left[0,1-h_{n}\right]
$$

Each $h_{n}$ defines a partition of $I^{\prime}=[0,1)$ into disjoint intervals $\left[(k-1) h_{n}, k h_{n}\right)$.

Using finite induction we shall prove for every fixed $n$ existence of functions $a_{i}^{n}: I^{\prime} \rightarrow A_{i}$, $i=1, \ldots, n$, and absolutely continuous $x_{n}: I^{\prime} \rightarrow \mathbb{R}^{d}$ for which:

$$
\begin{aligned}
& \left(a_{1}^{n}(t), \ldots, a_{n}^{n}(t)\right) \in C_{n}, a_{i}^{n}(\cdot) \text { constant on intervals }\left[(k-1) h_{i}, k h_{i}\right) ; \\
& x_{n}(0)=\xi ; \\
& \dot{x}_{n}(t)=u_{a_{1}^{n}(t), \ldots, a_{n}^{n}(t)}(t) \text { for } t \in I^{\prime} ; \\
& \left|x_{n}\left(k h_{i}\right)-a_{i}^{n}\left(k h_{i}\right)\right|<r_{i} / 2 \text { for } i=1, \ldots, n, k=0, \ldots, 1 / h_{i}-1 ; \\
& \left|x_{n}(t)-a_{i}^{n}(t)\right|<r_{i} \text { for } i=1, \ldots, n, t \in I^{\prime} .
\end{aligned}
$$

The first step consists in defining our functions on the interval $\left[0, h_{n}\right)$. We choose $a_{i} \in$ $A_{i}$, for $i=1, \ldots, n$, such that $\left|a_{i}-\xi\right|<r_{i} / 2$ and put $a_{i}^{n}(t)=a_{i}$ for $t \in\left[0, h_{n}\right)$. As $\left|a_{i}-a_{i-1}\right| \leq\left|a_{i}-\xi\right|+\left|\xi-a_{i-1}\right|<r_{i} / 2+r_{i-1} / 2<r_{i-1}$, so $\left(a_{1}^{n}(t), \ldots, a_{n}^{n}(t)\right) \in C_{n}$ and we may put

$$
x_{n}(t)=\xi+\int_{0}^{t} u_{a_{1}^{n}(\tau), \ldots, a_{n}^{n}(\tau)}(\tau) d \tau
$$

for $t \in\left[0, h_{n}\right)$. The conditions (14), (15), (16), (17) are satisfied due to our choice of the elements and (18) due to (13) and the inequalities $\left|x_{n}(t)-a_{i}^{n}(t)\right| \leq\left|x_{n}(t)-\xi\right|+\left|\xi-a_{i}^{n}(t)\right|<$ $r_{n} / 4+r_{i} / 2<r_{i}$.

Let now $1 \leq k<1 / h_{n}$ and suppose that all the elements have been defined for all intervals $\left[0, h_{n}\right), \ldots,\left[(k-1) h_{n}, k h_{n}\right)$. We put also $x_{n}\left(k h_{n}\right)=\lim _{t \rightarrow k h_{n}-} x_{n}(t)$.

There is a positive integer $j$ such that $h_{i} / h_{n} \in \mathbb{N}$ for $i=j, \ldots n$ and $h_{i} / h_{n} \notin \mathbb{N}$ for $i=1, \ldots, j-1$. If $j>1$ then for $i=1, \ldots, j-1$ we put $a_{i}^{n}(t)=a_{i}^{n}\left(m h_{i}\right)$ for $t \in\left[k h_{n},(k+1) h_{n}\right)$, where $m \in \mathbb{N}$ is such that $\left[(k-1) h_{n}, k h_{n}\right) \subset\left[m h_{i},(m+1) h_{i}\right)$. For every $i=j, \ldots, n$ we choose some $a_{i} \in A_{i}$ for which $\left|a_{i}-x_{n}\left(k h_{n}\right)\right|<r_{i} / 2$ and put $a_{i}^{n}(t)=a_{i}$ for $t \in\left[k h_{n},(k+1) h_{n}\right)$.

Obviously $\left|a_{i}^{n}(t)-a_{i-1}^{n}(t)\right|<r_{i-1}$ in $\left[k h_{n},(k+1) h_{n}\right)$ for $i=2, \ldots, j-1$, as the values did not change with respect to the previous interval. The same is true for $i=j+1, \ldots, n$ (if $j<n$ ) due to the choice of $a_{i}$-the same argument that we used at the first step works here. So we have to prove yet that $\left|a_{j}^{n}(t)-a_{j-1}^{n}(t)\right|<r_{j-1}$ (if $j>1$ ). For $m$ such that $k h_{n} \in\left(m h_{j-1},(m+1) h_{j-1}\right)$ we have

$$
\begin{aligned}
& \left|a_{j}^{n}(t)-a_{j-1}^{n}(t)\right|=\mid a_{j}-a_{j-1}^{n}\left(m h_{j-1} \mid\right. \\
& \leq\left|a_{j}-x_{n}\left(k h_{n}\right)\right|+\left|x_{n}\left(k h_{n}\right)-x_{n}\left(m h_{j-1}\right)\right|+\left|x_{n}\left(m h_{j-1}\right)-a_{j-1}^{n}\left(m h_{j}\right)\right| \\
& <r_{j} / 2+r_{j-1} / 4+r_{j-1} / 2<r_{j-1} .
\end{aligned}
$$

So $\left(a_{1}^{n}(t), \ldots, a_{n}^{n}(t)\right) \in C_{n}$ and $a_{i}^{n}(\cdot)$ are constant on $\left[k h_{n},(k+1) h_{n}\right)$. Now

$$
x_{n}(t)=x_{n}\left(k h_{n}\right)+\int_{k h_{n}}^{t} u_{a_{1}^{n}(\tau) \ldots a_{n}^{n}(\tau)}(\tau) d \tau \text { for } t \in\left(k h_{n},(k+1) h_{n}\right) .
$$

(14), (15), (16), (17) are satisfied on $\left[0,\left(k+1 h_{n}\right)\right.$. For $t \in\left[k h_{n},(k+1) h_{n}\right)$

$$
\left|x_{n}(t)-a_{i}^{n}(t)\right| \leq\left|x_{n}(t)-x_{n}\left(k h_{n}\right)\right|+\left|x_{n}\left(k h_{n}\right)-a_{i}^{n}\left(k h_{n}\right)\right|<r_{n} / 4+r_{i} / 2<r_{i}
$$


which proves (18) and completes the induction argument.

\section{The proof of the main theorem}

Due to (iii) for all $n$ the inequality $\left|\dot{x}_{n}(t)\right| \leq \lambda(t)$ holds in $I^{\prime}$ and thus $\dot{x}_{n}(\cdot)$ has a subsequence weakly convergent in $L^{1}$. All the properties of $r_{i}, s_{i}, a_{i}^{n}(\cdot), x_{n}(\cdot)$ that we assumed or proved are true also for any subsequence so we may consider that the sequence $\dot{x}_{n}(\cdot)$ is weakly convergent and in consequence $x_{n}(\cdot)$ is uniformly convergent to an absolutely continuous function $x_{0}(\cdot)$ whose derivative is the weak limit of $\dot{x}_{n}(\cdot)$. From (18) we see also that the sequence $a_{n}^{n}(\cdot)$ is uniformly convergent to $x_{0}(\cdot)$ and (16) implies the inclusion $\dot{x}_{n}(t) \in \hat{F}\left(t, a_{n}^{n}(t)\right)$ a.e. in $I^{\prime}$.

The standard argument based on Mazur's theorem shows that

$$
\dot{x}_{0}(t) \in \bigcap_{\varepsilon>0} \operatorname{clco} \bigcup\left\{\hat{F}(t, x):\left|x-x_{0}(t)\right|<\varepsilon\right\} \text { a.e. in } I^{\prime} .
$$

Due to (ii) the right-hand side above is contained in $F\left(t, x_{0}(t)\right)$ if this last set is convex. So we have to prove yet that $\dot{x}(t) \in F\left(t, x_{0}(t)\right)$ a.e. in the set $T_{0}=\{t \in I$ : $F\left(t, x_{0}(t)\right)$ is not convex $\}$. From Proposition 4 we deduce that $T_{0} \subset T=\left\{t \in I^{\prime}: x_{0}(t) \in\right.$ int $H(t)\}$. Obviously $T=\bigcup_{k \in \mathbb{N}} T_{1 / k}$ where $T_{s}=\left\{t \in I^{\prime}: x_{0}(t) \in H_{s}(t)\right\}$. (Remark that $T_{0}$ need not be measurable.)

Let us fix $s>0$. As $T_{s}=\operatorname{proj}_{I^{\prime}}\left(\operatorname{Gr}\left(H_{s}(\cdot)\right) \cap \operatorname{Gr}\left(x_{0}(\cdot)\right)\right)$ so Proposition 5 and the Projection Theorem ( [2], Thm. III.23) induce its measurability.

For any $n, p \in \mathbb{N}$ thanks to (12) we have

$$
\begin{aligned}
& \left|\dot{x}_{n+p}(t)-u_{a_{1}^{n+p}(t) \ldots a_{n}^{n+p}(t)}(t)\right|=\left|u_{a_{1}^{n+p}(t) \ldots a_{n+p}^{n+p}(t)}(t)-u_{a_{1}^{n+p}(t) \ldots a_{n}^{n+p}(t)}(t)\right| \\
& \quad \leq \sum_{i=n}^{n+p-1}\left|u_{a_{1}^{n+p}(t) \ldots a_{i+1}^{n+p}(t)}(t)-u_{a_{1}^{n+p}(t) \ldots a_{i}^{n+p}(t)}(t)\right| \leq \sum_{i=n}^{n+p-1} \alpha\left(t, r_{i}, a_{i}^{n+p}(t)\right) .
\end{aligned}
$$

We wish to apply in the above sum the inequality $\alpha\left(t, r_{i}, a_{i}^{n+p}(t)\right) \leq \eta\left(t, r_{i}, s_{i}\right)$ for $i \geq n$ and $t \in T_{s}$. This, in view of (7), requires the inclusion $a_{i}^{n+p}(t) \in H_{s_{i}}(t)$ for $t \in T_{s}$, equivalent to the inequality $d\left(a_{i}^{n+p}(t), H^{c}(t)\right) \geq 2 s_{i}$ which we shall prove to be true for sufficiently great $n$.

Fix $n_{1}$ such that $\left|x_{n}(t)-x_{0}(t)\right|<s$ for $n \geq n_{1}$ and all $t \in I^{\prime}$. For $t \in T_{s}, n \geq n_{1}$ and $n \leq i<n+p$ we have

$$
\begin{aligned}
2 s< & d\left(x_{0}(t), H^{c}(t)\right) \leq\left|x_{0}(t)-x_{n+p}(t)\right|+\left|x_{n+p}(t)-a_{i}^{n+p}(t)\right| \\
& +d\left(a_{i}^{n+p}(t), H^{c}(t)\right)<s+r_{i}+d\left(a_{i}^{n+p}(t), H^{c}(t)\right),
\end{aligned}
$$

so $d\left(a_{i}^{n+p}(t), H^{c}(t)\right)>s-r_{i}$. To have the desired inequality we need $s-r_{i} \geq 2 s_{i}$ that is $s \geq r_{i}+2 s_{i}$. As both $r_{i}$ and $s_{i}$ tend to zero there is $n_{2} \geq n_{1}$ such that if $i \geq n \geq n_{2}$ then this inequality is satisfied, so for any $n \geq n_{2}$ and $p \in \mathbb{N}$ we have for $t \in T_{s}$

$$
\left|\dot{x}_{n+p}(t)-u_{a_{1}^{n+p}(t) \ldots a_{n}^{n+p}(t)}(t)\right| \leq \sum_{i=n}^{n+p-1} \eta\left(t, r_{i}, s_{i}\right) .
$$

Let us fix now $\varepsilon>0$ and $r \geq n_{2}$ such that

$$
\sum_{k=r}^{\infty} \int_{0}^{1} \eta\left(t, r_{k}, s_{k}\right) d t<\varepsilon
$$


Then for any $m>r$ we have

$$
\int_{T_{s}}\left|\dot{x}_{m}(t)-u_{a_{1}^{m}(t) \ldots a_{r}^{m}(t)}(t)\right| d t<\varepsilon .
$$

For fixed $r$ the set of all functions $u_{a_{1}^{m}(\cdot) \ldots a_{r}^{m}(\cdot)}(\cdot)$ is finite for all $m>r$ because $a_{i}^{m}(\cdot)$ are constant on intervals $\left[(k-1) h_{i}, k h_{i}\right)$ and take values in finite sets $A_{i}$. This implies that the set of restrictions of $\dot{x}_{n}(\cdot)$ to $T_{s}$ (for all $n$ ) can be covered by a union of a finite family of balls with radius $\varepsilon$ in the space $L^{1}\left(T_{s}, \mathbb{R}^{d}\right)$ with metric derived from its norm.

The sequence $\left.\dot{x}_{n}\right|_{T_{s}}(\cdot)$ has thus a subsequence strongly convergent in $L^{1}$ and as it is already weakly convergent to $\left.\dot{x}_{0}\right|_{T_{s}}(\cdot)$ so the limits coincide. This subsequence has in turn a subsequence, say $\left.\dot{x}_{n_{l}}\right|_{T_{s}}(\cdot)$, convergent to $\left.\dot{x}_{0}\right|_{T_{s}}(\cdot)$ a.e. in $T_{s}$.

For $t \in T_{s}$ the multifunction $\hat{F}(t, \cdot)$ is continuous at $x_{0}(t)$, so $\dot{x}_{0}(t) \in \hat{F}\left(t, x_{0}(t)\right)$ because $\dot{x}_{n_{l}}(t) \in \hat{F}\left(t, a_{n_{l}}^{n_{l}}(t)\right)$ and $a_{n_{l}}^{n_{l}}(t) \rightarrow x_{0}(t)$ when $l \rightarrow \infty$. Taking into account the inclusion $\hat{F}\left(t, x_{0}(t)\right) \subset F\left(t, x_{0}(t)\right)$ we know now that $\dot{x}_{0}(t) \in F\left(t, x_{0}(t)\right)$ a.e. in $T_{s}$.

In view of the inclusion $T_{0} \subset \bigcup_{k=1}^{\infty} T_{1 / k}$ we may conclude that the set in which $\dot{x}_{0}(t) \notin$ $F\left(t, x_{0}(t)\right)$ is of measure zero. This completes the proof.

Note that the set $T_{0}$ need not be measurable but it is covered by a union of measurable sets $T_{1 / k}$.

\section{A few remarks on similar results}

There is quite a lot of results about the existence of solutions to differential inclusions for various types of solutions, under various assumptions and using various methods. A vast survey paper [11] containing almost 130 references was published in 1987 . The development continued later on also for solutions satisfying some additional requirements (like viability or invariance, see [1]) or for more general systems (like measure driven differential inclusions in [12]).

We mention here two particular papers dealing with mixed assumptions and related directly to Olech's Theorem - in both the continuity at $x$ in assumption (iv) of Theorem 1 was replaced by lower semicontinuity in a neighborhood of $x$.

First [8], where a Scorza-Dragoni type theorem was used and the existence of solutions proved with the use of the Kakutani Ky-Fan Fixed Point Theorem. Olech's result is there mentioned as a corollary - a similar method to deal with the lack of $\mathscr{L} \times \mathscr{B}$ measurability of $F$ was used as the one proposed by Olech in his unpublished sketch of correction of his proof.

Much later [4] was published where the approach by a fixed point theorem for multivalued maps with decomposable values in $L^{1}$ was applied (the $\mathscr{L} \times \mathscr{B}$-measurability of $F$ was required).

\section{Compliance with ethical standards}

Conflict of interest On behalf of all authors, the corresponding author states that there is no conflict of interest.

Open Access This article is distributed under the terms of the Creative Commons Attribution 4.0 International License (http://creativecommons.org/licenses/by/4.0/), which permits unrestricted use, distribution, and reproduction in any medium, provided you give appropriate credit to the original author(s) and the source, provide a link to the Creative Commons license, and indicate if changes were made. 


\section{References}

1. Aubin, J.-P.: Viability Theory. Systems and Control: Foundations and Applications. Birkhäuser, Boston (1991)

2. Castaing, C., Valadier, M.: Convex Analysis and Measurable Multifunctions. Lecture Notes in Mathematics, vol. 580. Springer, Berlin (1976)

3. Filippov, A.F.: On the existence of solutions of multivalued differential equations (in Russian). Mat. Zametki 10, 307-331 (1971)

4. Fryszkowski, A., Górniewicz, L.: Mixed semicontinuous mappings and their applications to differential inclusions. Set Valued Anal. 8, 203-217 (2000)

5. Hermes, H.: The generalized differential equation $\dot{x} \in R(t, x)$. Adv. Math. 4, 149-169 (1970)

6. Kaczyński, H., Olech, C.: Existence of solutions of orientor fields with non-convex right-hand side. Ann. Polon. Math. 29, 61-66 (1974)

7. Kuratowski, K., Ryll-Nardzewski, C.: A general theorem on selectors. Bull. Acad. Pol. Sci. 13, 397-403 (1965)

8. Łojasiewicz, S., Jr.: Some theorems of Scorza Dragoni type for multifunctions with application to the problem of existence of solutions for differential multivalued equations. In: Olech, C., Jakubczyk, B., Zabczyk, J. (eds.) Mathematical Control Theory, pp. 625-643. Banach Center Publ., vol. 14. PWN, Warsaw (1985)

9. Marchaud, A.: Sur les champs continus de demi-cônes convexes et leurs intégrales. Composit. Math. 3, 89-127 (1936)

10. Olech, C.: Existence of solutions of non-convex orientor fields. Boll. Unione Mat. Ital. 11, 3-11 (1975)

11. Schechter, E.: A survey of local existence theories for abstract nonlinear initial value problems. In: Gill, T.L., Zachary, W.W. (eds.) Nonlinear Simigroups, Partial Differential Equations and Attractors. Lecture Notes in Mathematics, vol. 1934, pp. 136-184. Springer, Berlin (1987)

12. Silva, G.N., Vinter, R.B.: Measure driven differential inclusions. J. Math. Anal. Appl. 202, 727-746 (1996)

13. Zaremba, S.K.: Sur une extension de la notion d'équation différentielle. C. R. Math. Acad. Sci. Paris 199, $12780-1280$ (1934)

Publisher's Note Springer Nature remains neutral with regard to jurisdictional claims in published maps and institutional affiliations. 